\title{
Aplicación del ajuste de Rietveld para correlacionar la evolución microestructural de fundiciones blancas con 18 y $25 \%$ en Cromo, templadas en aceite y sucesivos revenidos, con el comportamiento frente al desgaste abrasivo y esfuerzos de flexión
}

\author{
José F. Álvarez Antolín ${ }^{\mathrm{a}, \mathbb{}}$, Laura Francos Garrote ${ }^{\mathrm{a}}$, Juan Asensio Lozano ${ }^{\mathrm{b}}$ \\ ${ }^{a}$ Departamento de Ciencia de los Materiales e Ingeniería Metalúrgica, Edificio Departamental Este, Universidad de Oviedo, \\ c/ Wifredo Ricart s/n, 33204 Gijón, España \\ b Departamento de Ciencia de los Materiales e Ingeniería Metalúrgica, Escuela de Ingeniería de Minas, Energía y Materiales, \\ Universidad de Oviedo, c/ Independencia 13, 33004 Oviedo, España \\ Autor para la correspondencia: alvarezflorentino@uniovi.es
}

Enviado: 18 Julio 2017; Aceptado: 30 Noviembre 2017; Publicado on-Line:

\begin{abstract}
RESUMEN: Mediante la aplicación del método de afinamiento estructural de Rietveld se identificaron y cuantificaron las fases presentes en fundiciones blancas hipoeutécticas con 18 y $25 \%$ en Cromo, templadas en aceite y tras sucesivos revenidos a $500{ }^{\circ} \mathrm{C}$. Se correlacionaron los resultados con su comportamiento frente al desgaste abrasivo y bajo esfuerzos de flexión. En ambos casos el porcentaje de austenita retenida tras el temple resultó mínima. Durante los sucesivos revenidos, se constató en la calidad con 18\% en Cromo una redisolución parcial de los carburos $\mathrm{M}_{7} \mathrm{C}_{3}$ junto a una transformación entre carburos mixtos secundarios $\mathrm{M}_{7} \mathrm{C}_{3}$ y $\mathrm{M}_{2} \mathrm{C}$. La Martensita tras temple resultó un factor clave frente a la resistencia al desgaste abrasivo, resultando los revenidos tras el temple en aceite desfavorables para esta propiedad. La calidad con $25 \%$ en Cromo fue la que presentó un mayor contenido en martensita tras el temple, y por tanto la que mejor comportamiento presentó frente a este tipo de desgaste. Esta misma calidad, tras el doble revenido, fue la que mayor resistencia y capacidad de deformación alcanzó bajo esfuerzos a flexión. La fractura resultó frágil y mayoritariamente transgranular. Sin embargo, pudo observarse la presencia de zonas con fractura dúctil asociadas a martensita doblemente revenida.
\end{abstract}

PALABRAS CLAVE: Carburos secundarios; Desgaste abrasivo; Difracción de Rayos X; Fundición blanca altamente aleada en Cr; Método de Rietveld; Resistencia a flexión

Citar como/Citation: Álvarez Antolín, J.F.; Francos Garrote, L.; Asensio Lozano, J. (2018). "Aplicación del ajuste de Rietveld para correlacionar la evolución microestructural de fundiciones blancas con 18 y $25 \%$ en Cromo, templadas en aceite y sucesivos revenidos, con el comportamiento frente al desgaste abrasivo y esfuerzos de flexión". Rev. Metal. 54(1): e113. https://doi.org/10.3989/revmetalm.113

ABSTRACT: Application of Rietveld Refinement to the correlation of the microstructure evolution of white cast irons with 18 and $25 \%$-wt. Cr after oil quench and successive temper treatments, with abrasive wear and bending testing. Through the application of Rietveld's structural refinement method, the phases present in oil quenched and successively tempered treatments at $500{ }^{\circ} \mathrm{C}$ of hypoeutectic white cast irons containing 18 and $25 \%$-wt. $\mathrm{Cr}$ were identified. The former were correlated to the behavior shown under abrasive wear testing and bending testing too. The fraction of retained austenite after quenching for both highly alloyed irons was low. The alloy containing 18\%-wt. Cr showed the highest fraction of carbides after quenching, and yet it was observed that secondary carbides seemed to develop preferentially in former destabilized proeutectic austenite as compared to former destabilized eutectic austenite. On the other hand, the highly alloyed cast iron with $25 \%$-wt. Cr exhibited 


\begin{abstract}
a more homogeneous phase resulting from austenite transformation after tempering. It was also observed that the cast iron containing $18 \%$-wt. $\mathrm{Cr}$ showed partial solution of $\mathrm{M}_{7} \mathrm{C}_{3}$ carbides and a transformation of mixed secondary carbides of the $\mathrm{M}_{7} \mathrm{C}_{3}$ y $\mathrm{M}_{2} \mathrm{C}$ types. The fraction of martensite from quenching was detected to have a positive response to the resistance to abrasive wear, and illustrating of the inadequacy of quenching in oil for this sought property. The alloy bearing $25 \%$-wt. $\mathrm{Cr}$ was found to have the highest martensite fraction after quenching, and thus a higher abrasive wear resistance. After a double tempering treatment, under bending testing, it displayed the highest rupture stress and strain, yielding a fragile fracture with facets of the transgranular mode. It was however observed the presence of regions depicting ductile fracture associated to double tempered martensite.
\end{abstract}

KEYWORDS: Abrasive wear; Flexural strength; High chromium white cast iron; Rietveld refinement; Secondary carbides; X-ray Diffraction

ORCID: José F. Álvarez Antolín (https://orcid.org/0000-0003-2447-6575); Laura Francos Garrote (https://orcid. org/0000-0003-0484-9469); Juan Asensio Lozano (https://orcid.org/0000-0001-6552-2860)

Copyright: (C) 2018 CSIC. Este es un artículo de acceso abierto distribuido bajo los términos de la licencia de uso y distribución Creative Commons Reconocimiento 4.0 Internacional (CC BY 4.0).

\section{INTRODUCCIÓN}

Las fundiciones blancas altamente aleadas presentan un contenido en Cromo superior al $15 \%$. A partir de este porcentaje, su microestructura posee dos particularidades que condicionan sus propiedades. Una es que la fase matriz de su constituyente eutéctico es austenita por lo que resultan tenaces y mecanizables (Pero-Sanz, 1994). Para ello, en este último caso, deben someterse a un tratamiento isotérmico que transforme la austenita en perlita. Otra particularidad es que los carburos que forman parte de su eutéctica son carburos mixtos $(\mathrm{Fe}, \mathrm{Cr})_{7} \mathrm{C}_{3}$, llamados carburos $\mathrm{K}_{2}$, los cuales presentan una dureza entre 1200 y $1600 \mathrm{HV}$, superior a la de los carburos cementíticos (Fairhust y Rohrig, 1974). La gran templabilidad de su austenita permite que mediante un temple al aire se forme martensita. Si el temple se realiza en aceite el riesgo de agrietamiento aumenta, pero en cambio disminuye el porcentaje de austenita retenida. La transformación de la austenita en perlita tiene dos efectos negativos sobre el comportamiento en servicio de estas Fundiciones. Uno sobre la resistencia al desgaste, y otro sobre la tendencia de los carburos a ser arrancados de su matriz por el esfuerzo cortante que acompaña al desgaste abrasivo. Es por ello, que la mejor de las microestructuras es aquella en la que la fase matriz sea martensita (Fairhust y Rohrig, 1974). La austenita presenta una elevada templabilidad, debido a los elementos en solución sólida de sustitución, mayoritariamente Cromo, por lo que mediante un enfriamiento al aire se obtiene martensita. Sin embargo, la resistencia a la abrasión se ve mejorada si se favorece la precipitación de carburos secundarios, ricos en Cromo, uniformemente distribuidos en una matriz mayoritariamente martensítica, y si se ve reducido el porcentaje de austenita retenida (Gasan y Ertuk, 2013). Esto permitiría, a su vez, aumentar ligeramente la resistencia a la corrosión (Wiengmoon et al., 2011). La fracción en volumen de carburos eutécticos y la dureza macroscópica presenta una influencia poco significativa sobre la resistencia al desgaste (Scandian et al., 2009). Con temperaturas próximas a los $1000{ }^{\circ} \mathrm{C}$ y permanencias de $4 \mathrm{~h}$ se logra una desestabilización casi completa de la austenita (Powell y Bee, 1996). Estudios recientes han demostrado que adiciones de Boro, en el entorno de las 200 ppm, favorecen un aumento de los carburos secundarios al actuar este elemento como agente nucleante para la precipitación de dichos carburos (Bedolla-Jacuinde et al., 2016). Para minimizar los riesgos de agrietamiento es habitual realizar el enfriamiento al aire en calma, o mediante ventiladores. Sin embargo, un temple en aceite disminuiría el porcentaje de austenita retenida, aumentando el porcentaje de martensita, y por tanto, su teórica resistencia al desgaste. Las temperaturas de revenido habituales se sitúan en el rango $200-250^{\circ} \mathrm{C}$. Sin embargo, si el revenido se realizara a temperaturas más elevadas, entre 400 y $600^{\circ} \mathrm{C}$, sería posible lograr una segunda desestabilización de la austenita retenida, favoreciendo su transformación en nueva martensita (Davis, 1994). El contenido en austenita retenida aumenta si el porcentaje en Carbono de estas fundiciones también aumenta. Sin embargo, esta austenita se ve reducida mediante revenidos posteriores en el rango comprendido entre $450 \mathrm{y}$ $500{ }^{\circ} \mathrm{C}$ (Opapaiboon et al., 2015). La mayor dureza se alcanzaría con dobles revenidos en el entorno de los $500{ }^{\circ} \mathrm{C}$ (Fernández y Belzunce, 2006). Tiempos de permanencia elevados a estas temperaturas pueden originar la formación de carburos cementíticos, $\mathrm{K}_{\mathrm{c}}$, por transformación de estos carburos secundarios, disminuyendo de esta forma la resistencia al desgaste de la fundición (Wang et al., 2006), y su dureza (Wang et al., 2005). El Molibdeno en solución sólida, cuando su concentración supera el 3\%, tiende a favorecer la precipitación de carburos del tipo $\mathrm{M}_{2} \mathrm{C}$ y $\mathrm{M}_{6} \mathrm{C}$ durante revenidos a temperaturas cercanas a la crítica (Wiengmoon et al., 2011). A su vez, la presencia de Molibdeno disminuye 
las temperaturas de inicio de precipitación de la austenita proeutéctica y de comienzo de la reacción eutéctica: líquido $\rightarrow$ Austenita + carburos $\mathrm{K}_{2}$ (Tenorio et al., 2000).

Con el propósito de mejorar el comportamiento bajo condiciones de desgaste abrasivo de estas fundiciones blancas aleadas, y correlacionar éstas con su evolución microestructural, se tomaron dos muestras hipoeutécticas con 18 y $25 \%$ en Cromo que fueron sometidas a un tratamiento de desestabilización a $1000{ }^{\circ} \mathrm{C}$, durante $4 \mathrm{~h}$, con un posterior temple en aceite. Esta temperatura y tiempo de permanencia fueron seleccionados con los siguientes objetivos: 1) conseguir un elevado empobrecimiento en Cromo y Carbono de la austenita, y ocasionar un aumento de la temperatura $\mathrm{M}_{\mathrm{s}}$, lo cual permite reducir el riesgo teórico de agrietamiento durante dicho enfriamiento en aceite, 2) producir una disminución de la austenita retenida tras el temple en aceite, y 3) favorecer la precipitación de los carburos secundarios. Posteriormente fueron sometidas a un doble revenido a $500{ }^{\circ} \mathrm{C}$ con $8 \mathrm{~h}$ de permanencia. El motivo de que se seleccionara una permanencia elevada a esta temperatura fue el de examinar la posible precipitación de carburos cementíticos y su influencia sobre la resistencia al desgaste. Después de cada tratamiento, por aplicación del método de afinamiento de Rietveld, se analizó la evolución microestructural de ambas fundiciones, el contenido en austenita retenida, y los parámetros de red de las fases precipitadas. A su vez, se estudió la variación de la dureza, su comportamiento ante esfuerzos de flexión, y su resistencia bajo condiciones de desgaste abrasivo. En este trabajo se analiza la influencia del temple en aceite, de diferentes revenidos, y de la presencia de Mo en la composición química sobre la microestructura de estas fundiciones blancas. A su vez, se identifica la microestructura más apropiada para el comportamiento en servicio bajo condiciones de desgaste abrasivo y bajo esfuerzos a flexión. En particular, se investiga la influencia del Mo en la precipitación de carburos secundarios mediante la desestabilización de la austenita, y su correlación sobre el comportamiento bajo condiciones de desgaste abrasivo.

\section{MATERIALES Y MÉTODOS}

Se tomaron 2 muestras con las composiciones químicas señaladas en la Tabla 1 . Ambas muestras fueron tratadas $4 \mathrm{~h}$ a $1000{ }^{\circ} \mathrm{C}$ y enfriadas en aceite.

TABLA 1. Composición química de las calidades analizadas (\% en peso)

\begin{tabular}{lccccc}
\hline Calidad & C & Si & Mn & Cr & Mo \\
\hline $18 \% \mathrm{Cr}$ & 3,01 & 1,17 & 0,82 & 18,2 & 2,05 \\
$25 \% \mathrm{Cr}$ & 2,72 & 1,19 & 0,81 & 25,1 & 0,52 \\
\hline
\end{tabular}

Posteriormente fueron sometidas a un doble revenido de 8 h cada uno.

Mediante microscopía óptica y microscopía electrónica se analizó la evolución microestructural de ambas calidades Para la preparación de las muestras metalográficas, en el proceso de desbaste se emplearon lijas de $\mathrm{SiC}$, utilizando diferentes tamaños de grano abrasivo, desde grit 240 hasta 600. Durante el proceso de pulido se emplearon paños textiles sobre los que se extendió pasta de diamante de 6 y 1 micra. El reactivo de ataque empleado fue Nital 5, con un tiempo de suspensión de 6-10 segundos para la fundición con $18 \%$ en Cromo, y de 15-18 segundos para la fundición con $25 \%$ en Cromo. El microscopio óptico utilizado fue el modelo NIKON Epiphot 200, y las imágenes fueron obtenidas mediante el analizador de imagen Omnimet Enterprise. El microscopio electrónico empleado fue modelo JEOL JSM-5600.

Se midieron las durezas después de cada tratamiento térmico con un microdurómetro Vickers de la casa Buehler y modelo 1600-6100. La carga aplicada fue $0,5 \mathrm{~kg}$.

Mediante Difracción de Rayos X, con metal emisor de Molibdeno, se determinó el porcentaje y tipos de fases precipitadas. Los difractogramas se midieron en el equipo Seifert XRD 3000 T/T en configuración óptica Bragg-Brentano parafocal con rotación inversa y acoplada al detector. La radiación emitida por el tubo de Mo de foco fino, a una potencia de $40 \mathrm{kV}$ y $40 \mathrm{~mA}$, fue monocromatizada al doblete $\mathrm{K} \alpha: \lambda_{1}=0.709316 \AA$ y $\lambda_{2}=0.713607 \AA$. Los patrones se han registrado mediante un detector de centelleo $\mathrm{NaI}(\mathrm{Tl})$ midiendo el rango angular $2 \theta$. Éste se encontraba comprendido entre $12^{\circ}$ y $56^{\circ}$, con paso de $0,025^{\circ}$ y tiempo de contaje por paso de $30 \mathrm{~s}$. La determinación del porcentaje de fases cristalinas se ha establecido mediante el ajuste de dichos difractogramas utilizando el método de afinamiento estructural de Rietveld. Para ello, a partir del registro de las figuras de difracción obtenidas en ambas calidades, se realizó un afinamiento estructural empleando los ficheros de información cristalográfica presentes en la base de datos de estructuras inorgánicas ICSD (versión 2016) pertenecientes a las fases que, según la literatura técnica, podrían encontrarse precipitadas tras los diversos tratamientos térmicos realizados en ambas calidades (Fairhust y Rohrig, 1974; Pero-Sanz, 1994; Wang et al., 2006). Entre ellas, principalmente: Martensita $\left(\alpha^{\prime}\right)$, Austenita $(\gamma)$, carburos mixtos $\mathrm{Cr} / \mathrm{Fe}$ (en particular con estequimetrías $3: 2 ; 7: 3 ;$ y 23:6), y carburos mixtos $\mathrm{Mo}_{2} \mathrm{C}$ y $\mathrm{Fe}_{3} \mathrm{C}$. Como consecuencia, por aplicación del método de afinamiento estructural de Rietveld, se produce un ajuste de las figuras experimentales de difracción en base a las figuras de difracción de estas fases.

A su vez, se analizó la resistencia al desgaste abrasivo de todas las muestras mediante la proyección de 
partículas de corindón por medio de aire comprimido, según la norma ASTM G76 (2004), aplicando una presión de $200 \mathrm{MPa}$, un caudal de $20 \mathrm{~g} /$ minuto, y con un ángulo de incidencia sobre las superficies de las muestras de $70^{\circ}$. La respuesta del material se obtuvo pesando las muestras tras los siguientes intervalos de tiempo: 2, 4, 6, 8, y 10 min. Para cada intervalo de tiempo se empleó una muestra diferente del mismo material. Los ensayos de flexión se realizaron en una máquina INSTROM 5582, con célula de carga de $100 \mathrm{kN}$. Las probetas de flexión fueron de sección rectangular, con ancho $6 \mathrm{~mm}$ y espesor $4 \mathrm{~mm}$. La distancia entre apoyos fue de $36 \mathrm{~mm}$.

\section{RESULTADOS}

La Tabla 1 muestra las composiciones químicas de ambas fundiciones. Cabe destacar, además de las diferencias en Cromo, que la calidad con $18 \%$ en Cromo presenta un mayor contenido en Carbono y especialmente en Molibdeno. Este último favorece la templabilidad de la aleación y un endurecimiento secundario tras el revenido (Inthidech et al., 2012). La Fig. 1 muestra la microestructura obtenida para la calidad con $18 \%$ en Cromo tras los diferentes tratamientos térmicos. Las Figs. 1 (a y b) muestra la microestructura formada tras el temple en aceite. Ésta está formada por austenita proeutéctica y el constituyente eutéctico, donde la austenita formaría la fase matriz y los carburos eutécticos $\mathrm{K}_{2}$ la fase dispersa. Esta austenita se habría transformado mayoritariamente en martensita. Cabe destacar la presencia de carburos secundarios, precipitados principalmente en la austenita proeutéctica, mostrando ésta una coloración más oscura que la austenita del constituyente eutéctico. Esto pudiera deberse a la segregación del Mo hacia el líquido eutéctico favoreciendo un enriquecimiento de la asutenita eutéctica en este elemento, lo cual tiene un efecto inhibidor de la precipitación de carburos secundarios (Efremenko et al., 2013). La Fig. 1c muestra la microestructura tras el primer revenido y puede apreciarse como en este caso la distribución de los carburos secundarios resulta más homogénea. La Fig. 2 muestra la microestructura de la calidad con $25 \%$ en Cromo, la Fig. 2a tras el tratamiento del temple en aceite y la Fig. $2 b$ tras el primer revenido. Esta microestructura es muy similar a obtenida para la calidad con 18\% en Cromo. Sin embargo, en este caso, debe destacarse que la distribución de los carburos secundarios tras el tratamiento de temple resulta más homogénea que en el caso anterior. La Fig. 3 muestra la evolución de la distribución de estos carburos secundarios para la Fundición con 18\% en Cromo, desde el temple en aceite hasta su primer revenido. La Fig. 3a muestra que la precipitación de los carburos secundarios tras el temple se produce mayoritariamente en la (a)

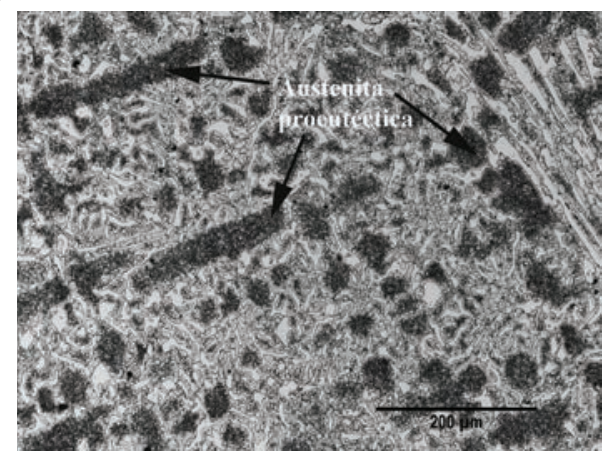

(b)

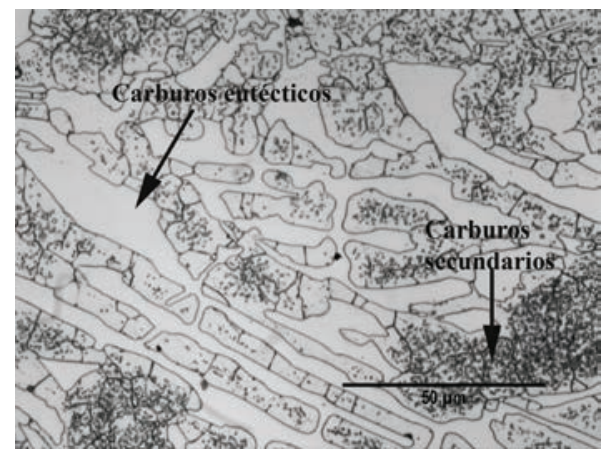

(c)

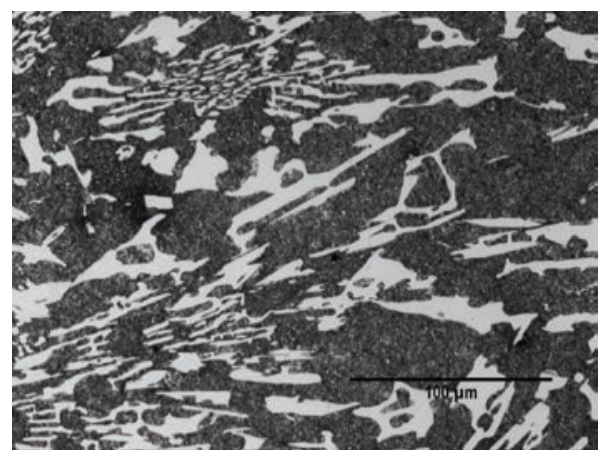

Figura 1. Evolución microestructural de la calidad con $18 \% \mathrm{Cr}$ : a) microestructura tras temple en aceite, 200X; b) microestructura tras temple en aceite, $1000 \mathrm{X}$; y c) microestructura tras temple en aceite y un revenido a $500{ }^{\circ} \mathrm{C}$ y $8 \mathrm{~h}, 500 \mathrm{X}$. 
(a)

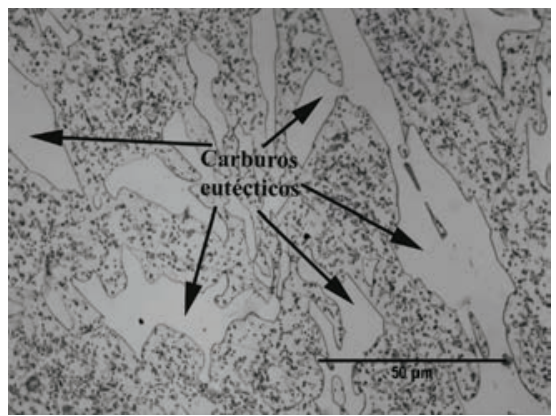

(b)

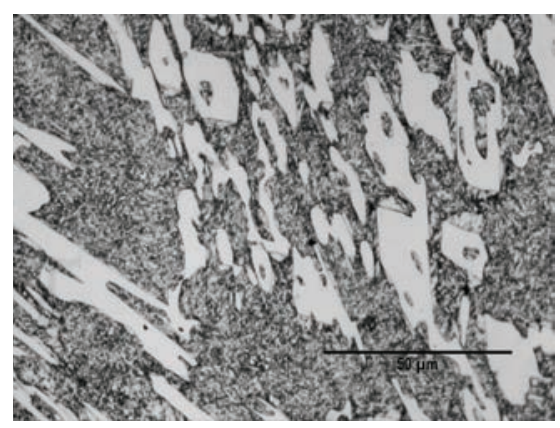

FIGURA 2. Evolución microestructural de la calidad con $25 \% \mathrm{Cr}$ : a) microestructura tras temple en aceite, 1000X; y b) microestructura tras temple en aceite y un revenido a $500{ }^{\circ} \mathrm{C}$ y $8 \mathrm{~h}, 1000 \mathrm{X}$.

(a)

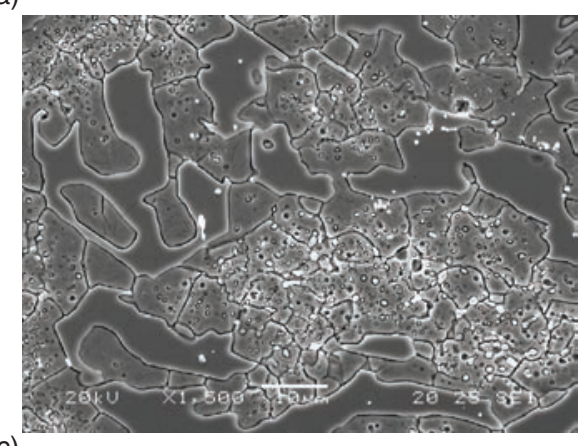

(c)

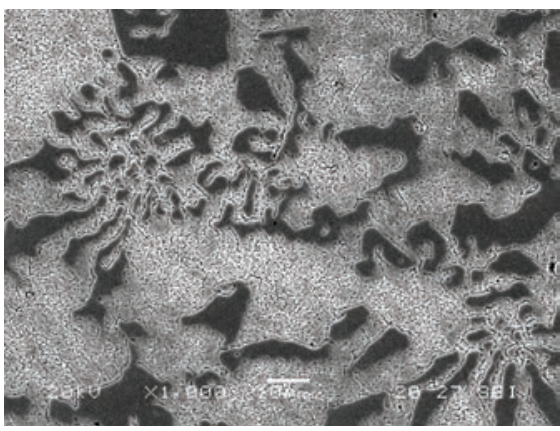

(b)

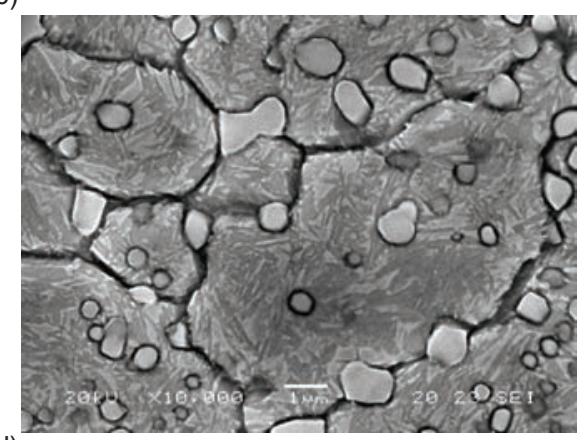

(d)

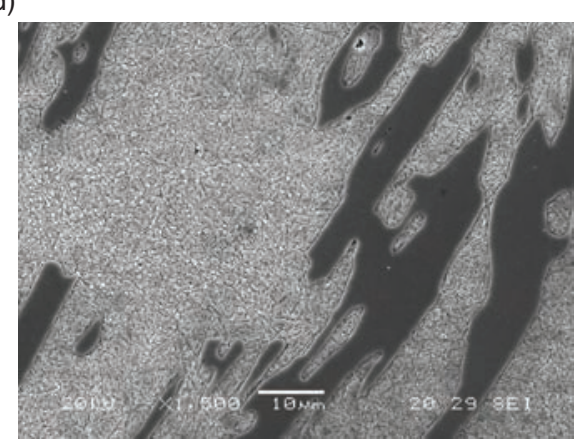

Figura 3. En imagen MEB, distribución de carburos secundarios para la calidad con $18 \%$ Cr: a) microestructura tras temple en aceite, 1500X; b) microestructura tras temple en aceite, 10000X; c) microestructura tras temple en aceite y un revenido a $500{ }^{\circ} \mathrm{C}$ y 8 h de mantenimiento, 1000X; d) microestructura tras temple en aceite y un doble revenido revenido a $500{ }^{\circ} \mathrm{C}$ y $8 \mathrm{~h}$ de mantenimiento, $1500 \mathrm{X}$.

austenita proeutéctica. La Fig. 3b muestra que ésta se produce tanto en las juntas de grano austenítico como en su interior. El diámetro de estos carburos es del orden de 1-2 micras. Las Figs. 3 (c - d) muestran una distribución más uniforme tras el primer y segundo revenido. La Fig. 4 muestra la distribución de carburos secundarios en la Fundición con $25 \%$ en Cromo. La Fig. 4a confirma una distribución más uniforme de los carburos secundarios entre la austenita proeutéctica y la eutéctica, y la Fig. $4 \mathrm{~b}$ parece indicar un aumento de la densidad de carburos secundarios tras el segundo revenido.

La Fig. 5 muestra los difractogramas obtenidos para ambas calidades. La Fig. 5a para la calidad con $18 \%$ en Cromo y la Fig. 5b para la calidad con $25 \%$ en Cromo. Para identificar las fases presentes y determinar sus porcentajes en peso se empleó el método de afinamiento estructural de Rietveld. La Fig. 6 muestra los ajustes Rietveld de las figuras experimentales de difracción (Hou et al., 2009). En las probetas analizadas del grupo con $18 \%$ en Cromo se observa que el temple estabiliza la martensita, que junto con el carburo $\mathrm{K}_{2}$, son las fases mayoritarias. También puede discernirse un pequeño porcentaje de austenita retenida. El primer revenido conduce a la redisolución parcial de carburos $\mathrm{K}_{2}$, muy probablemente porque el periodo de revenido resultó excesivo, y a la precipitación de carburos del tipo $\mathrm{M}_{2} \mathrm{C}$. 
(a)

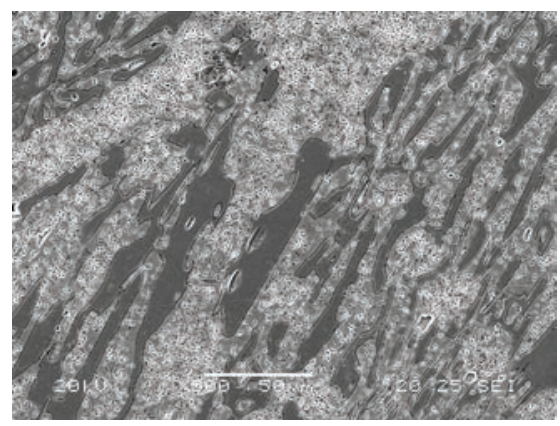

(b)

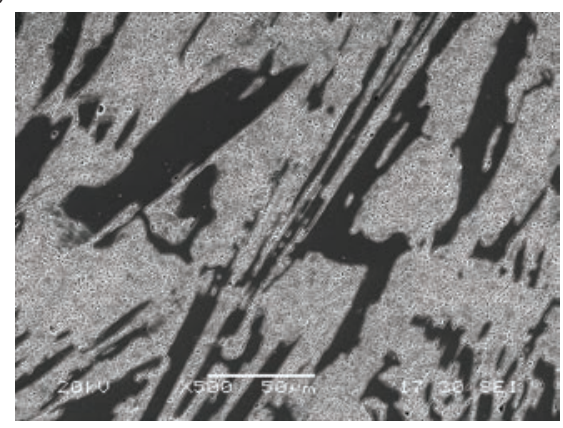

FIGURA 4. En imagen MEB, distribución de carburos secundarios para la calidad con $25 \% \mathrm{Cr}$ : a) microestructura tras temple en aceite, 500X; y b) microestructura tras temple en aceite y un doble revenido a $500{ }^{\circ} \mathrm{C}$ durante $8 \mathrm{~h}, 500 \mathrm{X}$.

(a)

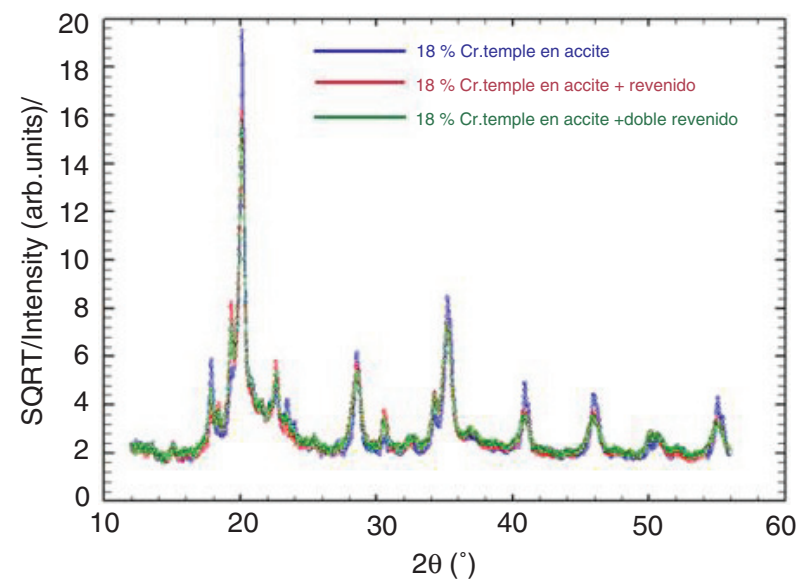

(b)

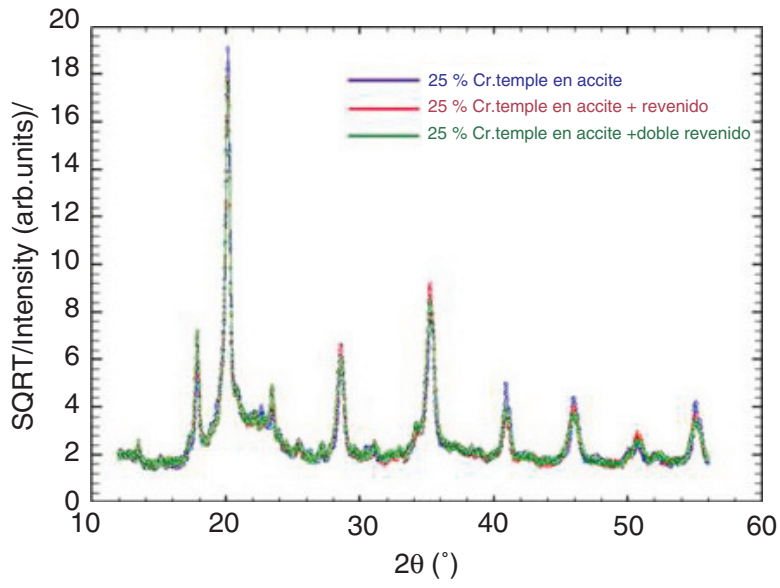

FIGURA 5. Difractogramas: a) difractogramas obtenidos para la calidad $18 \%$ en Cr; y b) difractogramas obtenidos para la calidad $25 \%$ en Cr.

Estos carburos presentan una mayor dureza que los carburos $\mathrm{K}_{2} .2200 \mathrm{HV}$ frente a $1600 \mathrm{HV}$. Sin embargo, durante el segundo revenido esta tendencia se invierte, precipitando nuevos carburos $\mathrm{K}_{2}$ y redisolviéndose parcialmente los carburos $\mathrm{M}_{2} \mathrm{C}$, posiblemente por el excesivo tiempo de permanencia a $500{ }^{\circ} \mathrm{C}$ durante este segundo revenido. Con respecto a las probetas del grupo con $25 \%$ en Cromo, los difractogramas demuestran que esta composición es más estable ante los distintos tratamientos, estando su microestructura constituida fundamentalmente por martensita y carburos $\mathrm{K}_{2}$. En este caso la austenita retenita tras el temple resulta ser mínima, y nula tras el primer revenido. Este hecho parece significar que la temperatura $\mathrm{M}_{\mathrm{s}}$ es ligeramente superior en la calidad con $25 \%$ en Cromo que en la de $18 \%$ Cromo, quizá debido a presentar esta última un contenido más elevado en Carbono. Las Tablas 2 y 3 muestran los parámetros microestructurales y distribuciones en peso de las fases identificadas en cada difractograma, tras los diferentes tratamientos térmicos a los que fueron sometidas estas calidades. De su análisis cabe destacar que:
1. La calidad con $18 \%$ en Cromo presenta tras el temple en aceite una proporción en peso de austenita retenida ligeramente superior a la de $25 \%$ en Cromo. Ambas fueron templadas en un medio de igual severidad, por lo que se deduce que el menor contenido en Carbono de esta segunda ha influido positivamente en la reducción de la austenita retenida. La calidad con $25 \%$ en $\mathrm{Cr}$ elimina la totalidad de austenita retenida tras el primer revenido.

2. Tras el primer revenido la Calidad con $18 \%$ en Cromo presenta una redisolución de carburos mixtos $\mathrm{K}_{2}$ y la precipitación de carburos mixtos $\mathrm{M}_{2} \mathrm{C}$. Sin embargo, esta tendencia se ve invertida en el segundo revenido. A su vez, la proporción en peso de carburos precipitados disminuye sensiblemente tras el primer revenido, para volver a aumentar tras el segundo revenido. Parece deducirse que los periodos de permanencia de $8 \mathrm{~h}$ durante los revenidos a $500{ }^{\circ} \mathrm{C}$ resultan excesivos para esta calidad, favoreciéndose una redisolución parcial de los carburos precipitados. La Fig. 7 muestra la 
(a)

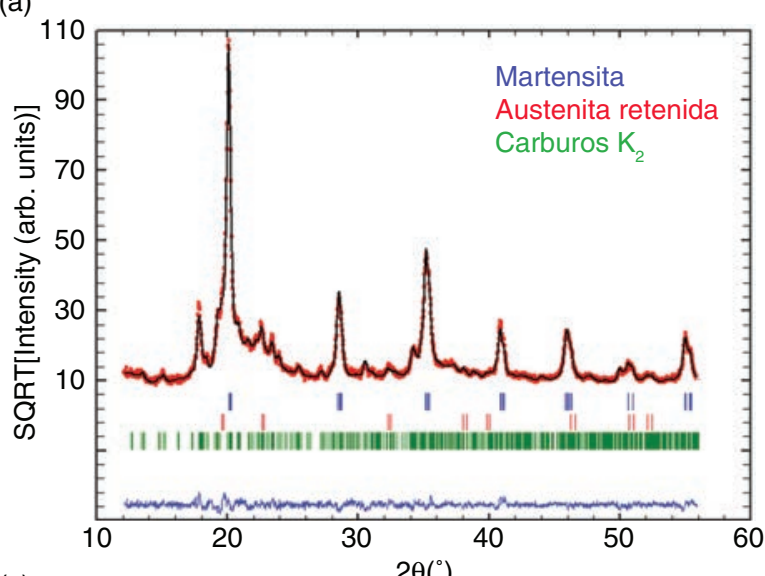

(c)
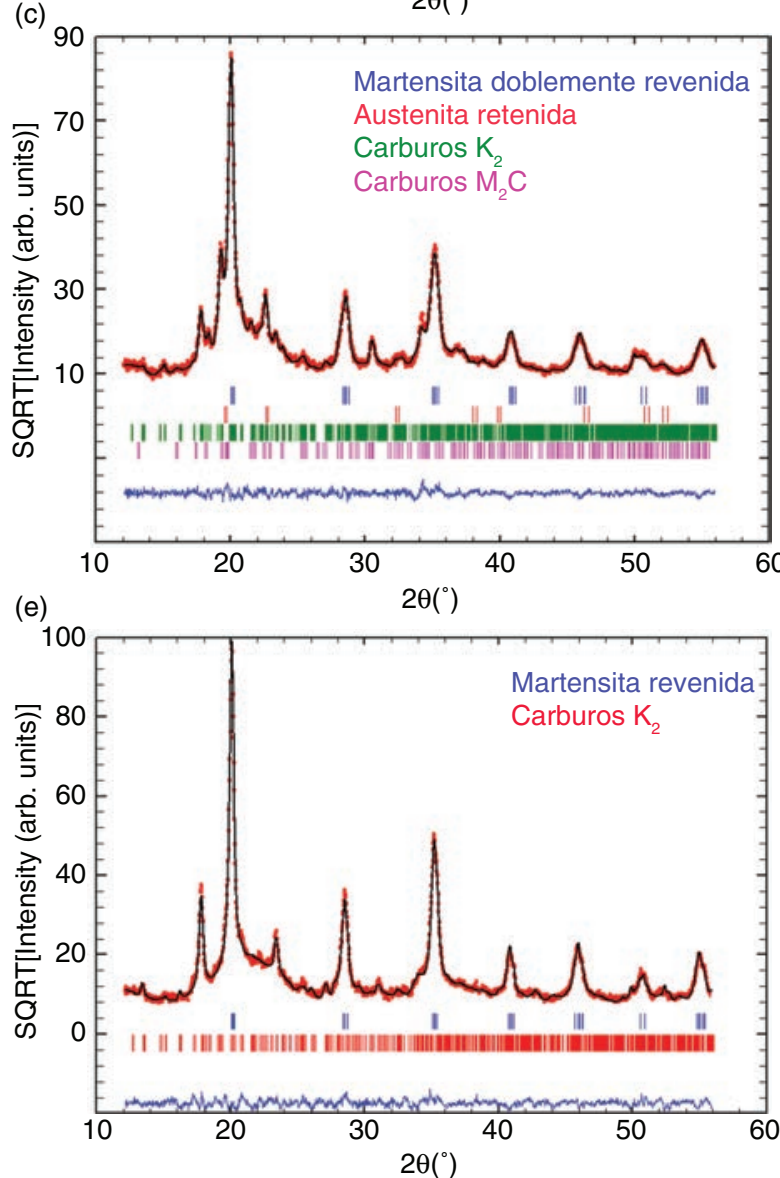

(b)

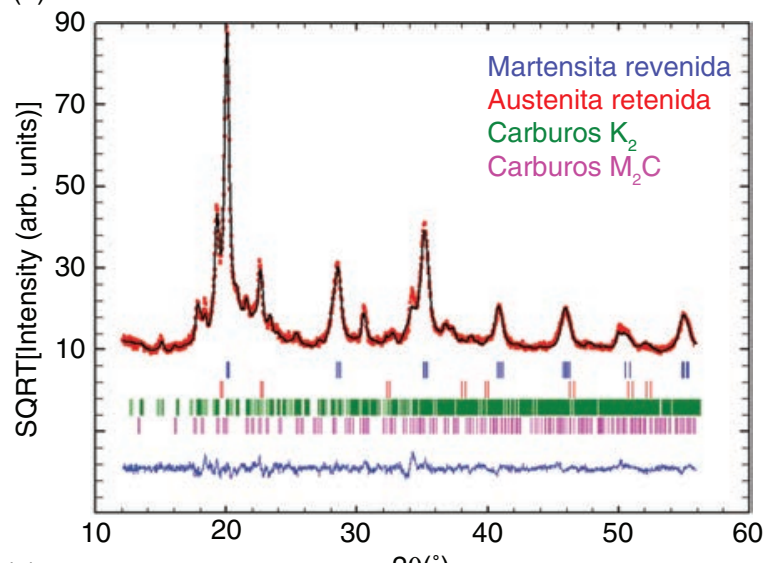

(d)

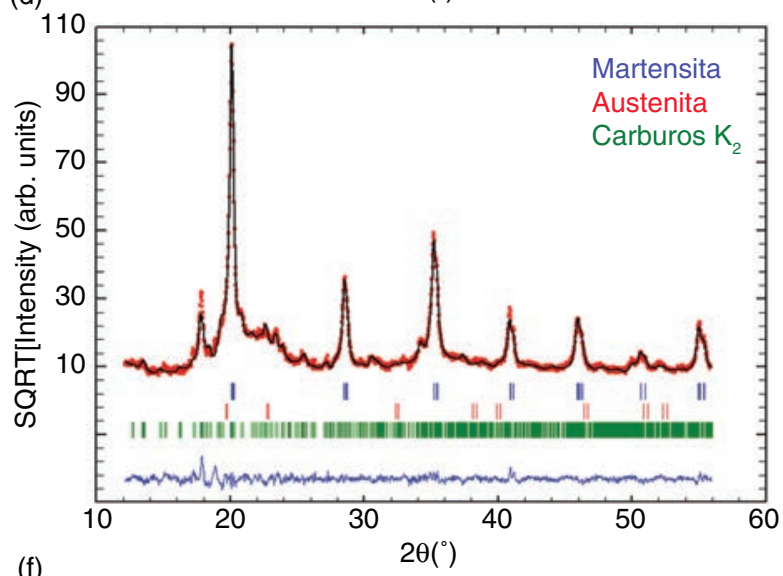

(f)

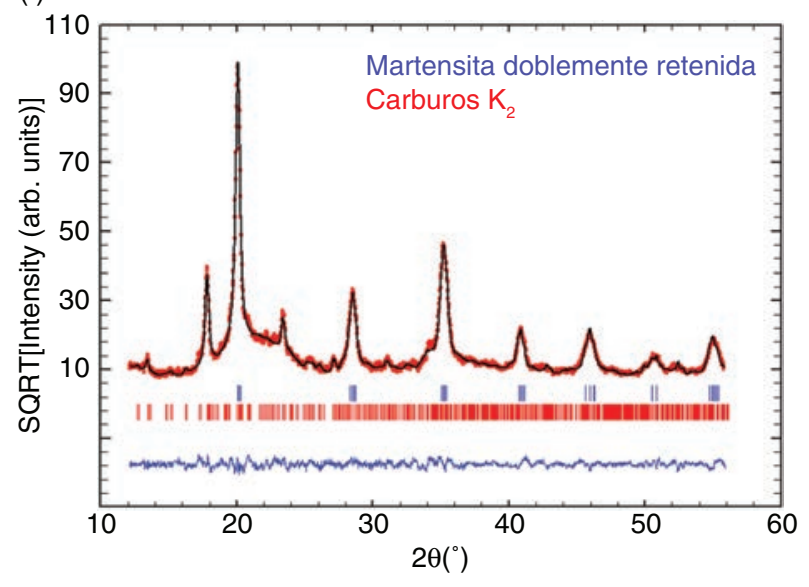

FiguRa 6. Afinamiento estructural de Rietveld sobre los difractogramas obtenidos: a) ajuste de Rietveld sobre la probeta con 18\% en Cr tras temple; b) sobre la probeta con $18 \%$ en Cr tras temple y un revenido; c) sobre la probeta con $18 \%$ en Cr tras temple y un doble revenido; d) sobre la probeta con $25 \%$ en $\mathrm{Cr}$ tras temple; e) sobre la probeta con $25 \%$ en $\mathrm{Cr}$ tras temple y un revenido; y f) sobre la probeta con $25 \%$ en Cr tras temple y un doble revenido.

presencia de estos carburos mixtos $\mathrm{M}_{2} \mathrm{C}$ tras los revenidos.

3. El comportamiento de la calidad con $25 \%$ en Cromo es más estable, produciéndose un aumento en la proporción de carburos tras los sucesivos revenidos por la precipitación de carburos secundarios.
La Tabla 4 muestra las durezas obtenidas, tomando 20 medidas en cada muestra. Como era de esperar, la calidad con 18\% en Cromo sufre un endurecimiento secundario tras el segundo revenido.

La Fig. 8 muestra los resultados obtenidos tras los ensayos de desgaste abrasivo. Se constata que las microestructuras obtenidas tras el temple en 
TABLA 2. Parámetros microestructurales y distribuciones en peso de las fases identificadas en cada difractograma, tras los diferentes tratamientos térmicos a los que fue sometida la calidad con $18 \%$ en $\mathrm{Cr}$

\begin{tabular}{|c|c|c|c|c|c|c|}
\hline Muestra & Ajuste Rietveld & Fases & a $(\AA)$ & b $(\AA)$ & c $(\AA)$ & $\%$ en peso \\
\hline \multirow{4}{*}{$18 O^{\mathrm{a}}$} & $\mathrm{R}_{\mathrm{wp}}=11,5$ & $\alpha^{\mathrm{b}}$ & 2.87113 & & 2.87887 & 58,4 \\
\hline & $\mathrm{Chi}^{2}=2,26$ & $\gamma^{\mathrm{b}}$ & 3.61768 & & & 2,6 \\
\hline & & $\mathrm{K}_{2}^{\mathrm{b}}$ & 4.51206 & 7.01843 & 12.10783 & 39,0 \\
\hline & & $\mathrm{M}_{2} \mathrm{C}^{\mathrm{b}}$ & & & & \\
\hline \multirow{4}{*}{$18 O^{\mathrm{a}}$} & $\mathrm{R}_{\mathrm{wp}}=12,3$ & $\alpha^{\mathrm{b}}$ & 2.87357 & & 2.88854 & 82,6 \\
\hline & $\mathrm{Chi}^{2}=2,48$ & $\gamma^{\mathrm{b}}$ & 3.61768 & & & 0,6 \\
\hline & & $\mathrm{K}_{2}^{\mathrm{b}}$ & 4.50851 & 7.01843 & 12.03998 & 8,7 \\
\hline & & $\mathrm{M}_{2} \mathrm{C}^{\mathrm{b}}$ & 2.91554 & & 3.87896 & 8,1 \\
\hline \multirow{4}{*}{18 OTT $^{\mathrm{a}}$} & $\mathrm{R}_{\mathrm{wp}}=10,4$ & $\alpha^{\mathrm{b}}$ & 2.87071 & & 2.89895 & 74,6 \\
\hline & $\mathrm{Chi}^{2}=1,62$ & $\gamma^{\mathrm{b}}$ & 3.61768 & & & 0,4 \\
\hline & & $\mathrm{K}_{2}^{\mathrm{b}}$ & 4.51110 & 7.02706 & 12.08683 & 20,6 \\
\hline & & $\mathrm{M}_{2} \mathrm{C}^{\mathrm{b}}$ & 2.91554 & & 3.87896 & 4,4 \\
\hline
\end{tabular}

${ }^{\text {a }} \mathrm{O}$ : temple en aceite: OT: temple en aceite + revenido; OTT: temple en aceite + doble revenido.

${ }^{\mathrm{b}}$ Todas las fases presentan una estructura cúbica salvo la $\mathrm{M}_{2} \mathrm{C}$ cuya estructura es hexagonal compacta.

TABLA 3. Parámetros microestructurales y distribuciones en peso de las fases identificadas en cada difractograma, tras los diferentes tratamientos térmicos a los que fue sometida la calidad con $25 \%$ en $\mathrm{Cr}$

\begin{tabular}{|c|c|c|c|c|c|c|}
\hline Muestra & Ajuste Rietveld & Fases & a $(\AA)$ & b (̊) & c ( $(\AA)$ & $\%$ en peso \\
\hline \multirow[t]{3}{*}{$25 \mathrm{O}^{\mathrm{a}}$} & $\mathrm{R}_{\mathrm{wp}}=14,3 \%$ & $\alpha^{\mathrm{b}}$ & 2.86986 & & 2.8802 & 77,2 \\
\hline & $\mathrm{Chi}^{2}=3,04$ & $\gamma^{\mathrm{b}}$ & 3.58502 & & & 1,1 \\
\hline & & $\mathrm{K}_{2}^{\mathrm{b}}$ & 4.50353 & 7.01027 & 12.11823 & 21,7 \\
\hline \multirow[t]{3}{*}{$25 O^{\mathrm{a}}$} & $\mathrm{R}_{\mathrm{wp}}=14,6 \%$ & $\alpha^{\text {b }}$ & 2.87114 & & 2.88662 & 44,7 \\
\hline & $\mathrm{Chi}^{2}=3,21$ & $\gamma^{\mathrm{b}}$ & & & & \\
\hline & & $\mathrm{K}_{2}^{\mathrm{b}}$ & 4.49248 & 6.98443 & 12.12621 & 55,3 \\
\hline \multirow[t]{3}{*}{25 OTT $^{\mathrm{a}}$} & $\mathrm{R}_{\mathrm{wp}}=13,7 \%$ & $\alpha^{\mathrm{b}}$ & 2.87015 & & 2.89153 & 43,0 \\
\hline & $\mathrm{Chi}^{2}=2,85$ & $\gamma^{\mathrm{b}}$ & & & & \\
\hline & & $\mathrm{K}_{2}{ }^{\mathrm{b}}$ & 4.49248 & 6.98443 & 12.12621 & 57,0 \\
\hline
\end{tabular}

${ }^{\text {a }}$ O: temple en aceite: OT: temple en aceite + revenido; OTT: temple en aceite + doble revenido.

b Todas las fases presentan una estructura cúbica.

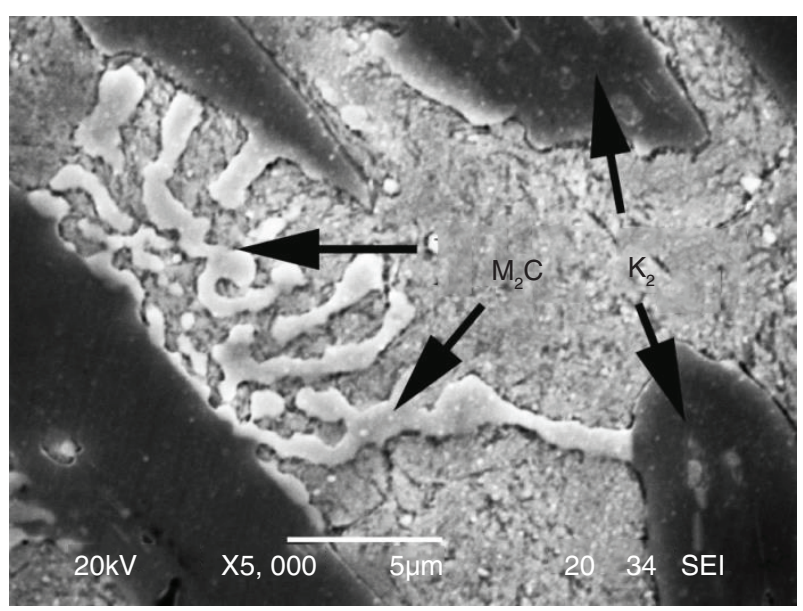

FIgURA 7. Carburos mixtos con Molibdeno del tipo $\mathrm{M}_{2} \mathrm{C}$, localizados en la calidad con $18 \%$ en $\mathrm{Cr}$ tras el doble revenido, $5000 \mathrm{X}$.
TABla 4. Microdurezas

\begin{tabular}{lcccccc}
\hline HV 0,5 & $\mathbf{1 8}$ & $\mathbf{1 8}$ & $\mathbf{1 8}$ & $\mathbf{2 5}$ & $\mathbf{2 5}$ & $\mathbf{2 5}$ \\
$\mathbf{O}^{\mathbf{a}}$ & OT $^{\mathbf{a}}$ & OTT $^{\mathbf{a}}$ & $\mathbf{O}^{\mathbf{a}}$ & OT $^{\mathbf{a}}$ & OTT $^{\mathbf{a}}$ \\
\hline $\mathrm{N}^{\text {o Medidas }}$ & 20 & 20 & 20 & 20 & 20 & 20 \\
Media & 825 & 708 & 780 & 804 & 650 & 570 \\
Mediana & 828 & 748 & 790 & 800 & 660 & 582 \\
$1^{\text {er }}$ cuartil & 816 & 685 & 770 & 780 & 608 & 577 \\
$3^{\text {er }}$ cuartil & 845 & 790 & 810 & 828 & 690 & 593 \\
\hline
\end{tabular}

${ }^{\text {a }}$ O: temple en aceite: OT: temple en aceite + revenido; OTT: temple en aceite + doble revenido

aceite son las que presentan una mayor resistencia frente al desgaste abrasivo. La calidad con un $25 \%$ en Cromo es la que presenta el mejor comportamiento. Ésta presenta un mayor porcentaje de martensita tras dicho temple, por lo que se deduce que 

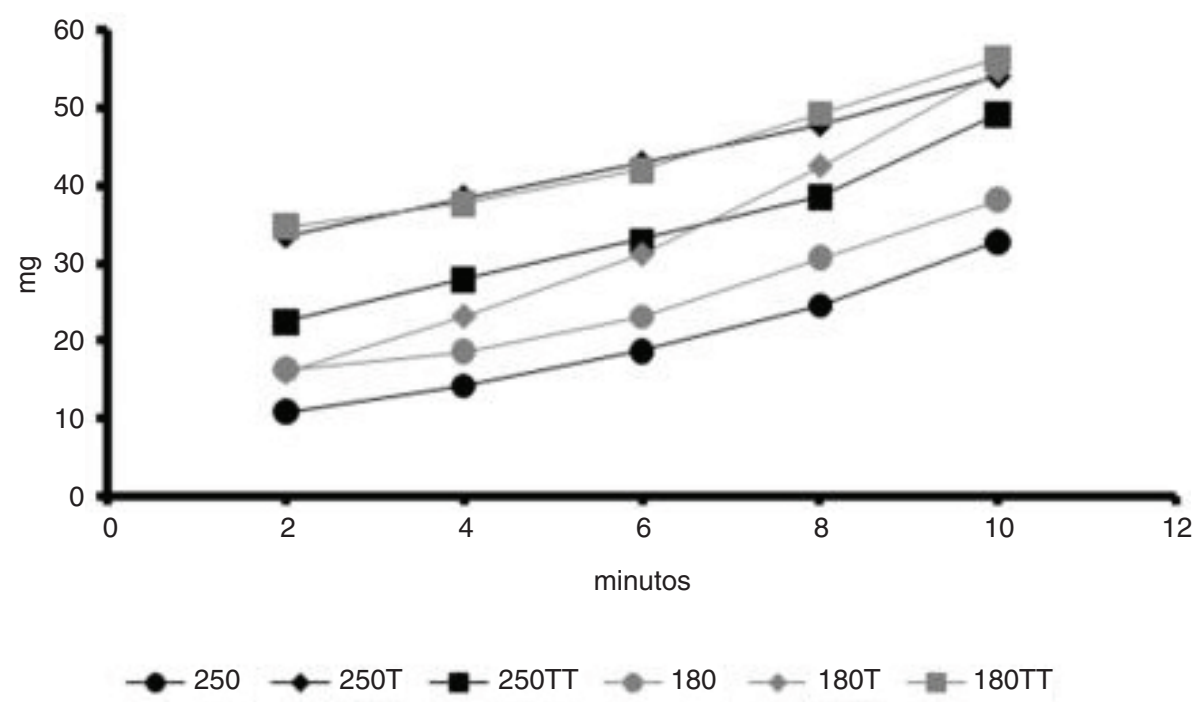

FIGURA 8. Resultados del ensayo de desgaste abrasivo (ASTM G76, 2004). O: Temple en aceite; OT: Temple en aceite + revenido; OTT: Temple en aceite + doble revenido.
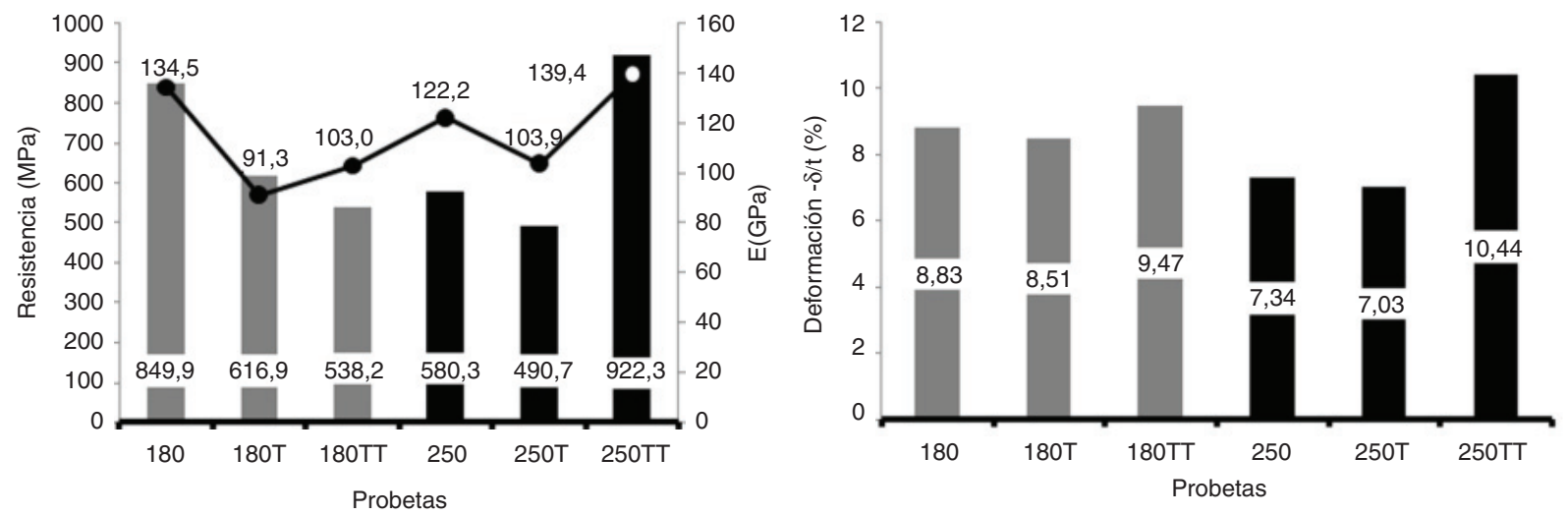

Figura 9. Ensayos de flexión en tres puntos: a) resistencia a flexión y módulo de elasticidad; y b) deformación a flexión medida como el cociente entre la flecha máxima $(\delta)$ y el espesor de la probeta $(\mathrm{t})$.

esta fase juega un papel relevante en la resistencia al desgaste abrasivo.

La Fig. 9 muestra los resultados obtenidos tras los ensayos de Flexión. Todas las roturas se produjeron sin deformación plástica. La Fig. 9a muestra los valores obtenidos de Resistencia y Rigidez (representada por el Módulo de Elasticidad). La Fig. 9b muestra la deformación obtenida en cada muestra, representada por el cociente entre la flecha máxima alcanzada y el espesor de la muestra. Cabe destacar que la calidad con $25 \%$ en Cromo tras los dos revenidos, a pesar de presentar la mayor proporción en peso total de carburos, fue la que alcanzó una mayor Resistencia, un mayor Módulo de Elasticidad, una mayor Deformación, y una menor Dureza. En ambas calidades se verificó una pérdida de rigidez y de capacidad de deformación tras el primer revenido, y su posterior recuperación tras el segundo revenido. Se analizaron los mecanismos de fractura de estas calidades. La Fig. 10 muestran unas fractografías obtenidas para la calidad con $25 \%$ en Cromo tras el temple en aceite, Fig. 10a, y tras el temple en aceite y doble revenido, Fig. 10b. La Fractura resulta frágil en ambos casos, y mayoritariamente transgranular. Sin embargo, puede observarse la presencia de áreas con fractura dúctil en la calidad con $25 \%$ en Cromo, lo cual justifica su mayor capacidad de deformación y su mayor resistencia a la fractura. Debe destacarse, que esta calidad es la que presentaba un mayor porcentaje en peso de martensita tras el temple en aceite, y por tanto, el doble revenido ha supuesto la precipitación de una gran cantidad de carburos secundarios de muy pequeño tamaño en una matriz de martensita doblemente revenida, que es la que ha generado áreas de fractura dúctil que han permitido elevar la 
(a)

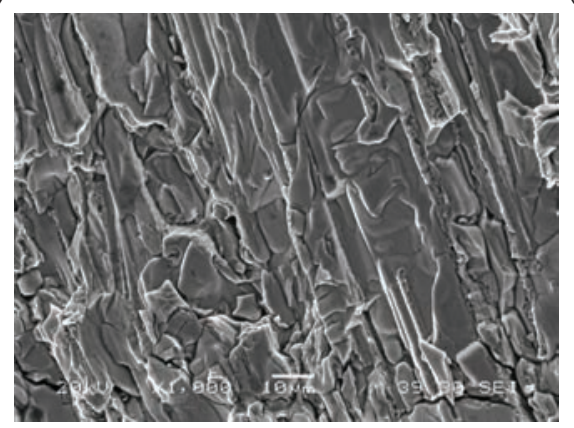

(b)

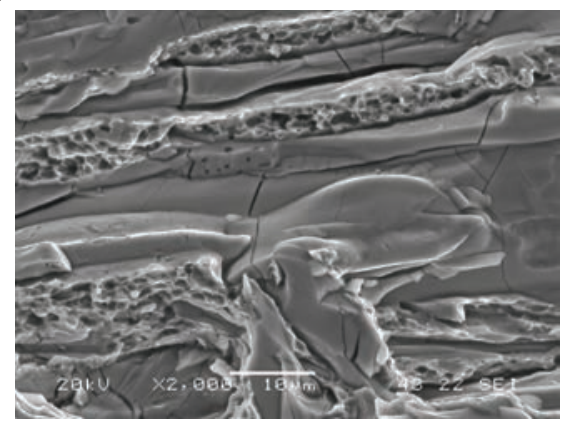

FIGURA 10. Facies de fractura resultante tras el ensayo de flexión: a) de la muestra con $25 \%$ en Cr templada en aceite, fractura frágil con desarrollo transgranular, 1000X; y b) de la muestra con 25\% en Cr templada en aceite más un doble revenido, 2000X.

capacidad de deformación y la fractura bajo mayores esfuerzos.

\section{CONCLUSIONES}

Por aplicación del método de afinamiento estructural de Rietveld se identificaron y ponderaron las fases presentes en fundiciones blancas hipoeutécticas con 18 y $25 \%$ en Cromo tras temple en aceite y tras sucesivos revenidos. Se correlacionaron los resultados con el comportamiento frente al desgaste abrasivo y esfuerzos de flexión, obteniéndose las siguientes conclusiones:

- La Fundición con 25\% en Cromo fue la que presentó un menor contenido en carburos y menor austenita retenida tras el temple en aceite. Tras los sucesivos revenidos continuó la precipitación de carburos secundarios, desapareciendo en su totalidad la austenita retenida.

- La Fundición con 18\% en Cromo presentó un porcentaje en carburos totales superior tras el temple, pero se advirtió una menor desestabilización en la austenita eutéctica respecto a la proeutéctica. Durante el primer revenido, se constató la precipitación de carburos del tipo $\mathrm{M}_{2} \mathrm{C}$. Tiempos de revenido excesivamente altos favorecen en esta calidad la redisolución parcial de los carburos precipitados.

- La mayor resistencia al desgaste abrasivo la presentaron microestructuras obtenidas tras el temple en aceite, lo que justifica el papel preponderante de la martensita sobre la resistencia al desgaste abrasivo. La calidad que presentó una mayor resistencia fue la que contenía un $25 \%$ en Cromo, que fue la que presentaba un mayor porcentaje en esta fase tras el temple en aceite. Los revenidos posteriores al temple en aceite no resultan favorables cuando se requiere resistencia al desgaste abrasivo.

- La mayor resistencia y capacidad de deformación bajo esfuerzos a flexión la alcanzó la calidad con $25 \%$ en Cromo tras el doble revenido, constatando la influencia significativa de la martensita doblemente revenida.

\section{REFERENCIAS}

ASTM G76 (2004). Standard Test Method for Conducting Erosion Tests by Solid Particle Impingement Using Gas Jets. ASTM International, West Conshohocken, PA, USA.

Bedolla-Jacuinde, A., Guerra, F.V., Mejía, I., Zuno-Silva, J., Maldonado, C. (2016). Boron effect on precipitation of secondary carbides during destabilization of high chromium white iron. Int. J. Cast. Metal. Res. 29 (1-2), 55-61. https://doi.org/10.1080/13640461.2016.1142236.

Davis, J.R. (1994). ASM Specialty Handbook - Cast Irons. ASM International, Kinsman Road, Ohio, USA.

Efremenko, V., Shimizu, K., Chabak, Y. (2013). Effect of Destabilizing Heat Treatment on Solid-State Phase Transformation in High-Chromium Cast Irons. Metall. Mater. Trans. A. 44 (12), 5434-5446. https://doi.org/10.1007/ s11661-013-1890-9.

Fairhust, W., Rohrig, K. (1974). Abrasion resistant high chromium cast irons. Foundry Trade Journal 136, 685-698.

Fernández, I., Belzunce, F.J. (2006). Influencia de diversos tratamientos térmicos en la microestructura de una fundición blanca con alto contenido en cromo. Rev. Metal. 42 (4), 279-286. https://doi.org/10.3989/revmetalm.2006.v42. i4. 27.

Gasan, H., Ertuk, F. (2013). Effects of a destabilization heat treatment on the microstructure and abrasive wear behavior of high chromium white cast iron investigated using different characterization techniques. Metall. Mater. Trans. A. 44 (11), 4993-5005. https://doi.org/10.1007/ s11661-013-1851-3

Hou, Q.Y., Huang, Z.Y., Wang, J.T. (2009). Application of rietveld refinement to investigate the high chromium white cast iron austempered at different temperatures. J. Iron Steel Res. Int. 16 (4), 33-38. https://doi.org/10.1016/ S1006-706X(09)60057-1.

Inthidech, S., Sricharoenchai, P., Matsubara, Y. (2012). Effect of molybdenum content on subcritical heat treatment behaviour of hypoeutectic 16 and $26 \mathrm{wt}-\%$ chromium cast irons. Int. J. Cast. Metal. Res. 25 (5), 257-263. https://doi.org/10. 1179/1743133612Y.0000000009.

Opapaiboon, J., Sricharoenchai, P., Inthidech, S., Matsubara, Y. (2015). Effect of carbon content on heat treatment behavior of multi-alloyed white cast iron for abrasive wear resistance. Mater. Trans. 56 (5), 720-725. https://doi. org/10.2320/matertrans.M2015001.

Pero-Sanz, J.A. (1994). Materiales para Ingeniería. Fundiciones Férreas. Editor Dossat, Madrid.

Powell, G.L.F., Bee, J.V. (1996). Secondary carbide precipitation in an $18 \mathrm{wt} \%$ Cr-1 wt \% Mo white iron. J. Mater. Sci. 31 (3), 707-711. https://doi.org/10.1007/BF00367889. 
Scandian, C., Boher, C., de Mello, J.D.B., Rezai-Aria, F. (2009). Effect of molybdenum and chromium contents in sliding wear of high-chromium white cast iron: The relationship between microstructure and wear. Wear 267 (1-4), 401-408. https://doi.org/10.1016/j.wear.2008.12.095.

Tenorio, J.A.S., Albertin, E., Espinosa, D.C.R. (2000). Effects of Mo additions on the solidification of high chromium cast iron. Int. J. Cast. Metal. Res. 13 (2), 99-105. https://doi.org $10.1080 / 13640461.2000 .11819393$

Wang, J., Zuo, R.L., Sun, Z.P., Li, C., Liu, H.H., Yang, H.S., Shen, B.L., Huang, S.J. (2005). Influence of secondary carbides precipitation and transformation on hardening behavior of a $15 \mathrm{Cr}-1 \mathrm{Mo}-1.5 \mathrm{~V}$ white iron. Mater.
Charact. 55 (3), 234-240. https://doi.org/10.1016/j. matchar.2005.06.002

Wang, J., Sun, Z., Shen, B., Gao, S., Zuo, R., Li, C., Huang, S. (2006). Effects of secondary carbide precipitation and transformation on abrasion resistance of the $16 \mathrm{Cr}-1 \mathrm{Mo}-$ $1 \mathrm{Cu}$ white iron. J. Mater. Eng. Perform. 15 (3), 316-319. https://doi.org/10.1361/105994906X108602.

Wiengmoon, A., Pearce, J.T.H., Chairuangsri, T. (2011). Relationship between microstructure, hardness and corrosión resistance in $20 \mathrm{wt} . \% \mathrm{Cr}, 27 \mathrm{wt} . \% \mathrm{Cr}$ and $36 \mathrm{wt} . \% \mathrm{Cr}$ high chromium cast irons. Mater. Chem. Phys. 125 (3), 739-748. https://doi.org/10.1016/j.matchemphys.2010. 09.064 . 